\title{
Ultrasound for Critical Care Physicians: The Pleura and the Answers that Lie Within
}

\author{
Heidi L. Erickson MD \\ Division of Pulmonary, Critical Care and Occupational Medicine \\ University of lowa Hospitals and Clinics \\ lowa City, IA
}

A 67-year-old woman with a 40-pack-year smoking history was admitted to the intensive care unit with acute respiratory failure secondary to adult respiratory distress syndrome (ARDS) in the setting of pneumococcal bacteremia. On admission, she required endotracheal intubation and vasopressor support. She was ventilated using a low tidal volume strategy and was relatively easy to oxygenate with a PEEP of 5 and $40 \%$ FiO2. After 48 hours of clinical improvement, the patient developed sudden onset tachypnea and increased peak and plateau airway pressures. A bedside ultrasound was subsequently performed (Figures 1 and 2).

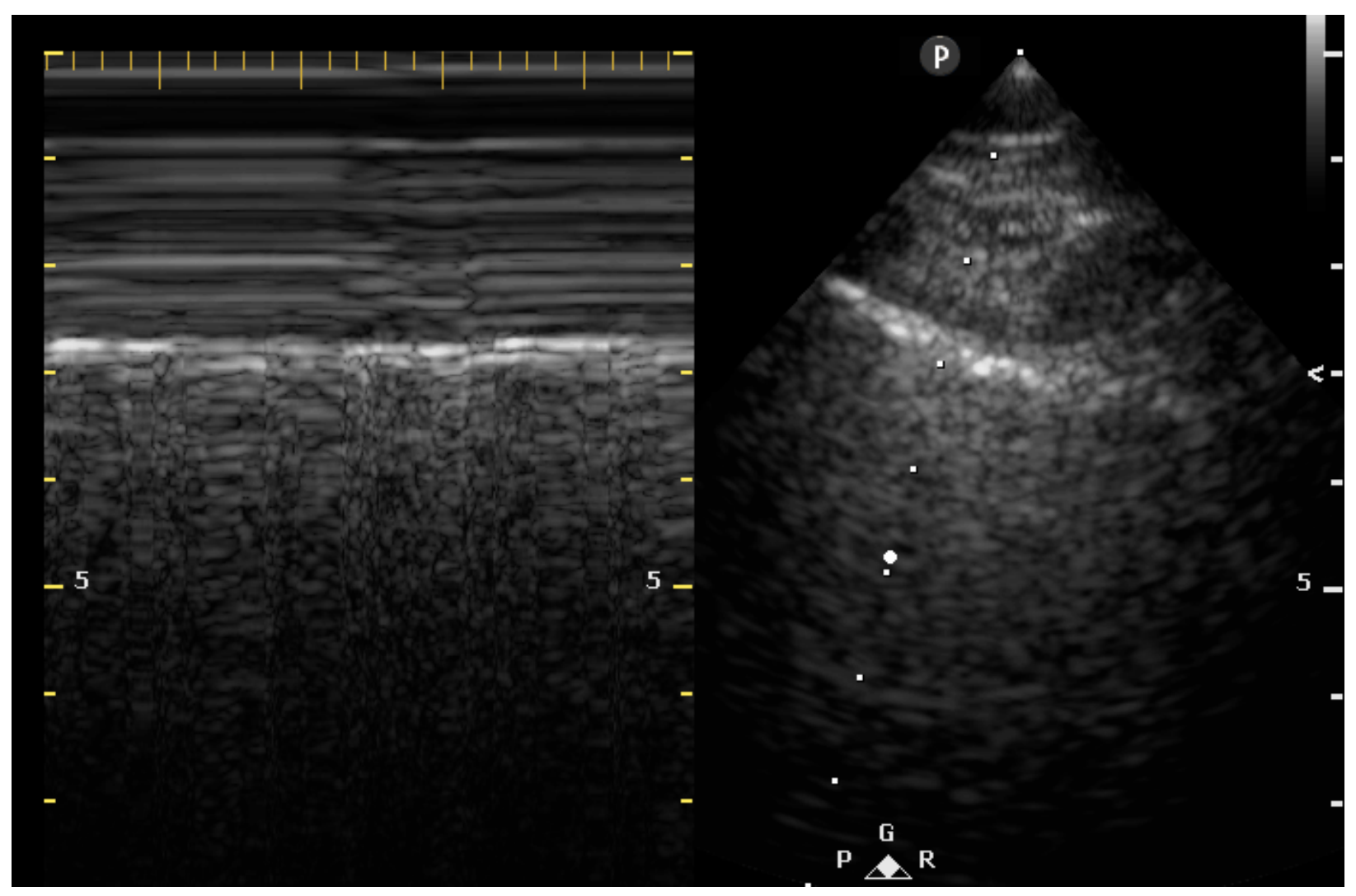

Figure 1. Two- dimensional ultrasound image of the right lung with associated M-mode image. 


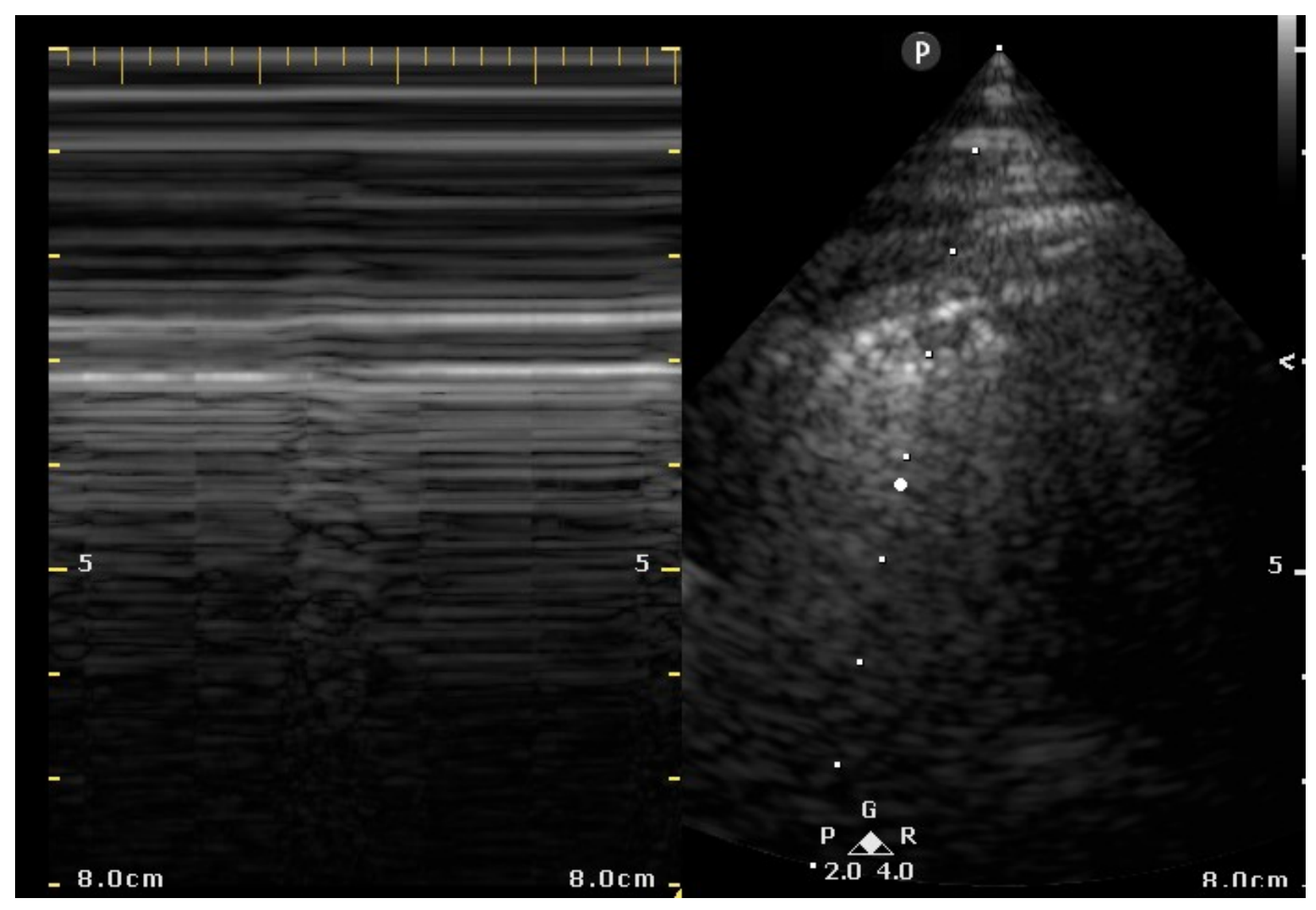

Figure 2. Two- dimensional ultrasound image of the left lung with associated M-mode image.

What is the cause of this patient's acute respiratory decompensation and increased airway pressures?

1. Pericardial effusion

2. Pneumothorax

3. Pulmonary edema

4. Pulmonary embolism 


\section{Correct! \\ 2. Pneumothorax (left sided)}

Figure 1 demonstrates a normal lung surface with the presence of lung sliding and the seashore sign under M-mode (Figure 3).

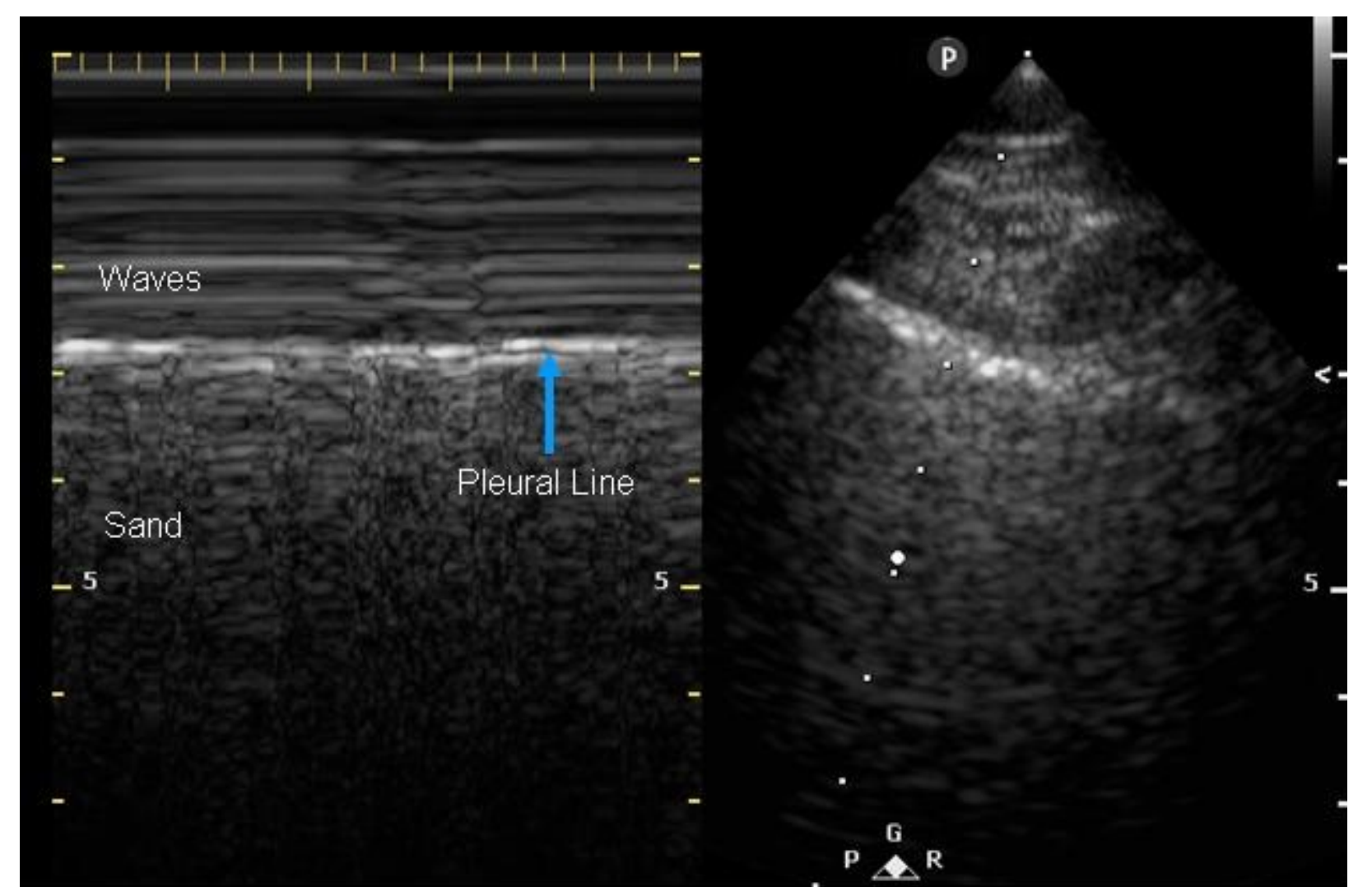

Figure 3. Two- dimensional ultrasound image of the right lung with seashore sign. Lung sliding is dynamic, identified as the horizontal movement along the pleural line, and is best seen at the apex. M-mode, which detects motion over time, provides further proof that lung sliding is present (1).

In Figure 2, lung sliding is absent and M-mode reveals a stratosphere or barcode sign, a pattern of parallel horizontal lines above and below the pleura highlighting its lack of movement. In this clinical context, this is indicative of a pneumothorax (Figure 4). 


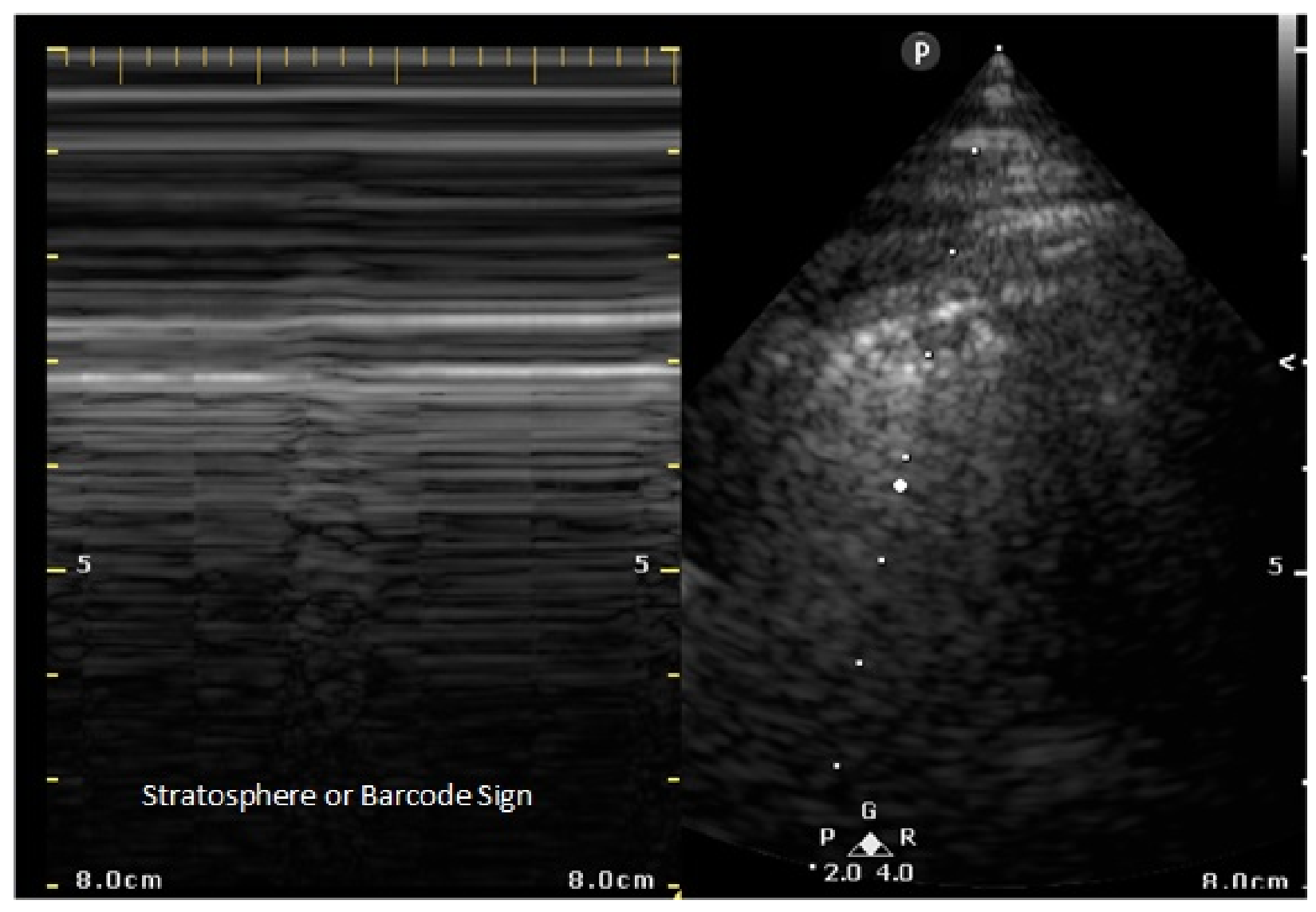

Figure 4. Two- dimensional ultrasound image of the left lung with stratosphere or barcode sign.

Lung ultrasound in the critically ill is established as a rapid and reliable technique to evaluate a variety of thoracic diseases. Ultrasound has been shown to be superior to CXR for diagnosing pneumothoraces and, in some studies, as sensitive as CT scan. While the negative predictive value for lung sliding is nearly $100 \%$, the specificity is reported to range from $60-99 \%$ depending on the patient and clinical context. As seen with pneumothoraces, lung sliding is also attenuated in ARDS, pulmonary fibrosis, pleural adhesions, atelectasis, and phrenic nerve paralysis. 2 In conclusion, lung ultrasound is useful in the rapid detection of pneumothoraces especially in those who are unstable and/or mechanically ventilated.

\section{References}

1. Lichtenstein DA. Lung ultrasound in the critically ill. Ann Intensive Care. 2014;4(1):1. [CrossRef] [PubMed]

2. Husain LF, Hagopian L, Wayman D, Baker WE, Carmody KA. Sonographic diagnosis of pneumothorax. J Emerg Trauma Shock. 2012;5(1):76-81. [CrossRef] [PubMed] 